\title{
Ultrasound-Guided Selective Pulsed Radiofrequency Treatment of Great Auricular Nerve for Post-Herpetic Neuralgia of the Head and Neck: A Case Report
}

\author{
Hong-Su Zhou' \\ Ting-Ting $\mathrm{Li}^{2}$ \\ $\mathrm{Yu} \mathrm{Pi}^{3}$ \\ Song Wen ${ }^{4}$ \\ Ting-Hua Wang (1D) \\ Liu-Lin Xiong' \\ 'Department of Anesthesiology, Affiliated \\ Hospital of Zunyi Medical University, \\ Zunyi, Guizhou, 563000, People's \\ Republic of China; ${ }^{2}$ Editorial Department \\ of Ibrain Journal, Affiliated Hospital of \\ Zunyi Medical University, Zunyi, Guizhou, \\ 563000, People's Republic of China; \\ ${ }^{3}$ National Traditional Chinese Medicine \\ Clinical Research Base and Western \\ Medicine Translational Medicine Research \\ Center, South West Medical University, \\ Luzhou, 646000, People's Republic of \\ China; ${ }^{4}$ Pain Management, Affiliated \\ Hospital of Zunyi Medical University, \\ Zunyi, Guizhou, 563000, People's \\ Republic of China
}

Objective: The current study aimed to further verify the feasibility of ultrasound-guided selective pulsed radiofrequency (PRF) therapy of greater auricular nerve (GAN) in the treatment of head and neck post-herpetic neuralgia (PHN) by observing the efficacy and safety.

Methods: Under the guidance of high-frequency ultrasound (Frequency: $10 \mathrm{MHz}$ ), the GAN was identified by a radiofrequency electrode trocar with a transverse in-plane approach, which was inserted into the GAN, then the inner needle of the trocar was retracted. After adjusting the technical variables (electrode tip temperature: $42^{\circ} \mathrm{C}$, output voltage: $60 \mathrm{~V}$, pulse frequency: $2 \mathrm{~Hz}$, pulse width: $22 \mathrm{~ms}$, single duration: $240 \mathrm{~s}$, two times), the radiofrequency electrode placed on the auricle and below the ear for sensory and motor tests began to work. Results: The pain in the left head and neck of the patient lasted for more than 1 month, we decided to try selective PRF of GAN guided by ultrasound for the first time. Immediately after the treatment, 11-point pain intensity numerical rating scale (PI-NRS) score ranged from 5 to 1 . During his hospitalization, mecobalamine and gabapentin were taken instead of opioids. Seven days after the procedure, PI-NRS score was 2, the degree of numbness the patient he felt by himself in the original lesion area relieved from $100 \%$ to $40 \%$, the depression module of the Patient Health Questionnaire-9 (PHQ-9) score was from 7 to 5, the Generalized Anxiety Disorder-7 (GAD-7) score from 8 to 4, the range of pain areas was reduced to external auditory tract, and there were no adverse events occurring.

Conclusion: The ultrasound-guided selective PRF treatment of GAN was safe and effective in the improvement of PHN in the head and neck, which is worthy of clinical promotion.

Keywords: post-herpetic neuralgia, great auricular nerve, pulsed radiofrequency

\section{Background}

Department of Anesthesiology, Affiliated Hospital of Zunyi Medical University, No. 20I. Da Lian Road, Hui Chuan District,

Zunyi, Guizhou, 563000, People's

Republic of China

Email4994650I0@qq.com

Ting-Hua Wang

Department of Anesthesiology, Affiliated Hospital of Zunyi Medical University, No. 20I. Da Lian Road, Hui Chuan District,

Zunyi, Guizhou, 563000, People's

Republic of China

Email Wangth_email@I63.com
Post-herpetic neuralgia (PHN) is the most common complication of herpes zoster, which is the pain lasting for 1 month or more after the healing of the herpes zoster rash. ${ }^{1}$ However, some scholars also have believed that the such pain lasting for more than 3 months is named as PHN. ${ }^{2}$ Clinically, PHN is characterized by central or deafferentation pain with persistent burning or paroxysmal tingling and resistance to peripheral destructive procedures. ${ }^{3}$ Commonly, the pain from PHN persists for many months or even years, which causes mental disorders, interferes with sleep and activities of daily living. The treatment methods of PHN mainly include drug therapies and physical therapies. In recent years, pulsed radiofrequency (PRF) and great 
auricular nerve (GAN) block technology have been gradually and widely used for the treatment of PHN and great auricular neuralgia respectively. ${ }^{4-6}$ However, to our knowledge, there were few related reports describing corresponding results when GAN is in the administration of PRF all over the world. Thus, we reported for the first time a case of a patient treating with selective PRF treatment of GAN under the ultrasound-guided due to PHN in the head and neck.

\section{Case Report Medical History}

A 55-year-old man presented with burning pain and scattered red papules, followed by colorless transparent blisters and faded gradually blisters in the left head and neck, with paroxysmal attack and irregular less than 1 month ago. He was admitted to a local hospital and diagnosed with herpes zoster and herpetic neuralgia. At that time, the specific pain area was in the left auricle, behind the ear, occipitalia and neck (Figure 1A). The physicians in his local hospital adopted an accepted and effective therapy measures including famciclovir, pregabalin and mecobalamin for acute herpes zoster and herpetic neuralgia. Despite the skin lesion healed, the pain in the skin lesions seemed to be more and more severe. An 11-point pain intensity numerical rating scale (PI-NRS) (to be used to measure pain intensity, where $0=$ no pain and $10=$ worst possible pain, Which in the treatment and management of chronic pain, it plays an important role for various degrees of global improvement. ${ }^{7}$ ) was 8 . And routine oral drugs, including pregabalin (50 mg-75 mg ter in die (tid)) and mecobalamin ( $0.5 \mathrm{mg}$ tid), became less and less effective, and even when experienced crushing pain, he had to rely on opioids (tramadol $50 \mathrm{mg}-100 \mathrm{mg}$ ) to ease and sleep. Based on drastic changes in the patient's pain intensity for more than a month, PRF ablation of left $\mathrm{C} 2$ dorsal ganglion and left superficial cervical nerves was performed with the hlep of digital substraction angiography (DSA) after diagnosed of PHN (left C2-4). It was regrettable that the patient seemed to be unable to clearly remember whether or not he had taken other medication orally and performed the physicotherapeutics before the PRF. We could only obtain useful information mentioned the above from the summary from his hospital as discharged. Fortunately, pain symptom was improved, and the pain area was also reduced, mainly concentrating on the auricle and part of the external ear (Figure 1B), except for complete numbness he felt in the left head and neck. He was discharged with a PI-NRS score of 5 at onset and took medicines (gabapentin: $0.3 \mathrm{mg}$ tid, mecobalamin: $0.5 \mathrm{mg}$ tid, tramadol: $50 \mathrm{mg}-100 \mathrm{mg}$ taking or adding it when required) after 3 days (d) postoperatively. On the 12th d after PHN treatment, the patient felt pain similar to acupuncture and electric shock in the left auricle and part of the external ear, despite increasing the dose of tramadol, he was so tortured, which seriously affected his mood and sleep quality. Then, he was transferred to our pain department. IP-NRS score was 5, somatic self-rating scale (SSS) score was 41, the depression module of the Patient Health Questionnaire (PHQ-9) score was 7, Generalized Anxiety Disorder - 7 (GAD - 7) score was 8. Vital signs were stable, and there were no obvious pathological changes in heart, lung, abdomen and other physical examinations and some routine biochemical examinations were normal.
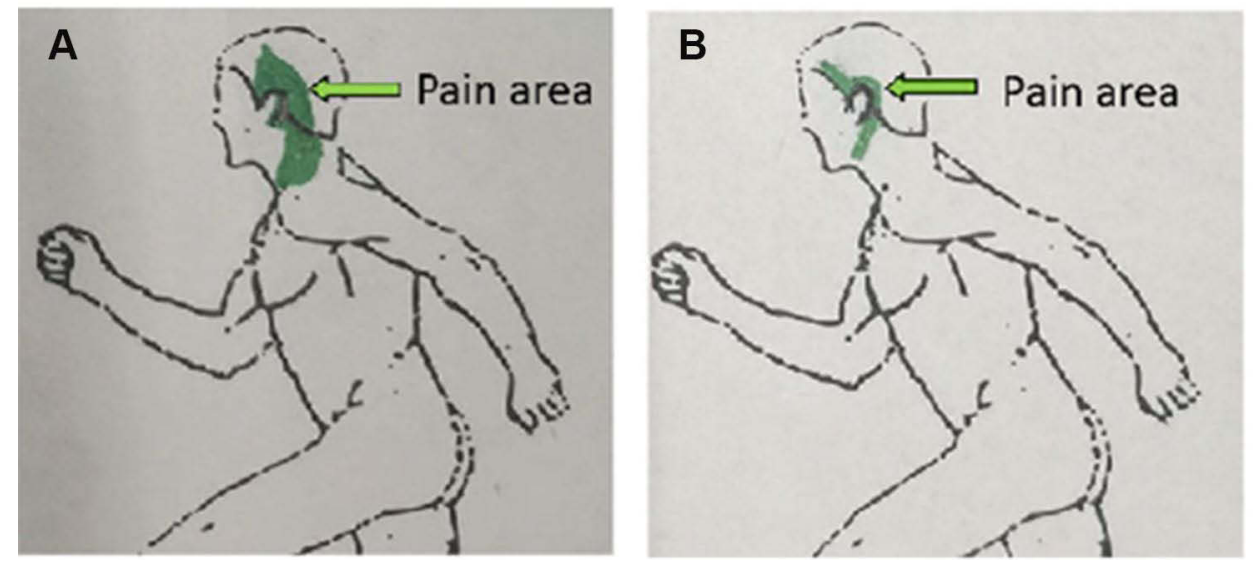

Figure I The pain area changes of the patient. (A) Pain area before all treatments. (B) Pain area after the first PRF treatment (C2 dorsal ganglion and left superficial cervical nerves). 
Among them, the SSS was designed by Mao Jialiang of the Department of Cardiology, Shanghai Renji Hospital (Shanghai, China), it includes 20 items (scored from 1-4, $1=$ "not at all" to 4="every day") that cover somatic, anxiety, depression and mixed anxiety-depression symptoms. The score for mixed anxiety-depression symptoms is based on the individual anxiety and depression scores. ${ }^{8}$ The total scores of 30, 40, and 60 representing cut-off points for mild, moderate, and severe somatization disorders in the SSS. A score of 36 or 37 was used as a cut-off score indicating "positive" for screening of clinical somatization in the Diagnostic and Statistical Manual and Mental Fifth Edition (DSM-V). The GAD-7 was developed in the USA as an valuable screening tools for detecting anxiety disorders in primary care patients, and the PHQ-9 measures both the presence and severity of the nine symptoms of major depression in the DSM-IV. ${ }^{9,10}$ Each item is marked on a $0-3$ scale involving the frequency of anxiety manifestations over the last two weeks $(0=$ "not at all or little" to 4="nearly every day") in the GAD-7, total scores of 5, 10, and 15 represent mild, moderate, and severe levels of anxiety. ${ }^{11}$ Similarly, the total scores of 5, 10, 15, and 20 representing cut-off points for mild, moderate, moderatesevere and severe depression in the PHQ-9. A score of 10 was used as a cut-off score, regardless of for the GAD-7 and the PHQ-9, indicating "positive" for screening of clinical depression and GAD, ${ }^{11}$ and they have shown good reliability and validity in previous studies. ${ }^{8-11}$

\section{Treatments \& Outcomes}

In line with the effect of the previous treatment, we have considered that the pain area was mainly in the innervation area of the GAN, and oral medicines were less effective. In addition, the effect of GAN block might be transient, and we had knew that early PRF in patients with

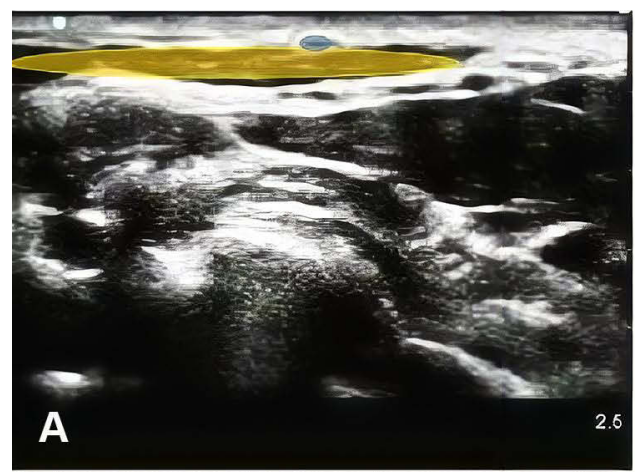

intractable PHN was likely to bring a satisfying therapeutic effect and prognosis. ${ }^{12}$ Therefore, with development and application of a new technology, GAN block in auriculotemporal surgery under the ultasound-guided in our institute, ultrasound-guided selective PRF treatment of GAN was planned. The patient took the right supine position in the treatment room, whose vital sign were monitored with continued electrocardiograph (ECG) and the venous channel was established. Under the guidance of high - frequency ultrasound (Frequency: $10 \mathrm{MHz}$ ), the GAN was identified by a $22 \mathrm{G} * 10 \mathrm{~cm} * 5 \mathrm{~mm}$ radiofrequency electrode trocar with a transverse in-plane approach (Figure 2A and B), which was inserted into the GAN, and then the inner needle of the trocar was retracted. Figure 2B showed the complete route of the radiofrequency needle entering the body and the location of the tip to the GAN. Before the radiofrequency electrode placed on the auricle and below the ear for sensory and motor tests began to work, adjusting the technical variables (electrode tip temperature: $42^{\circ} \mathrm{C}$, output voltage: 60 $\mathrm{V}$, pulse frequency: $2 \mathrm{~Hz}$, pulse width: $22 \mathrm{~ms}$, single duration: $240 \mathrm{~s}$, two times) and retracting the inner needle in the radiofrequency electrode trocar. During the treatment, paroxysmal needling sensation occurred in the auricle and neck under the ear. With the finish of radiofrequency, the electrode was pulled out. Subsequently, confirming the patient was safe and satisfied, and transferred to the ward.

Immediately after PRF treatment, the patient's NRS score was 1 , and mecobalamin ( $0.5 \mathrm{mg}$ tid) and gabapentin $(0.3 \mathrm{mg}$ tid) were took to consolidate effect. When he was discharged on $7 \mathrm{~d}$ after surgery, the NRS score was 2 , the pain scope was narrowed and concentrated on the external auditory canal screen (Figure 3), the numbness (when he regarded degree of numbness at admission as $100 \%$ ) was relieved to $40 \%$, the

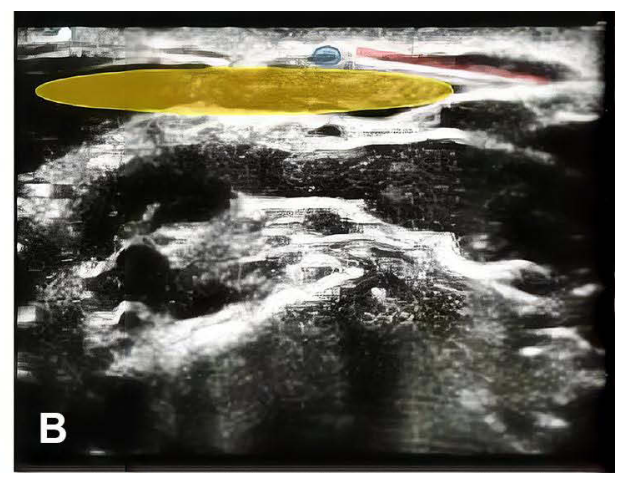

Figure 2 The radiofrequency ultrasound images of the GAN puncture. (A) The yellow area represented the sternocleidomastoid (SCM) muscle, and the blue area represented the GAN. (B) The yellow area represented the SCM muscle, the blue area represented the GAN, and the red area represented the radiofrequency needle. 


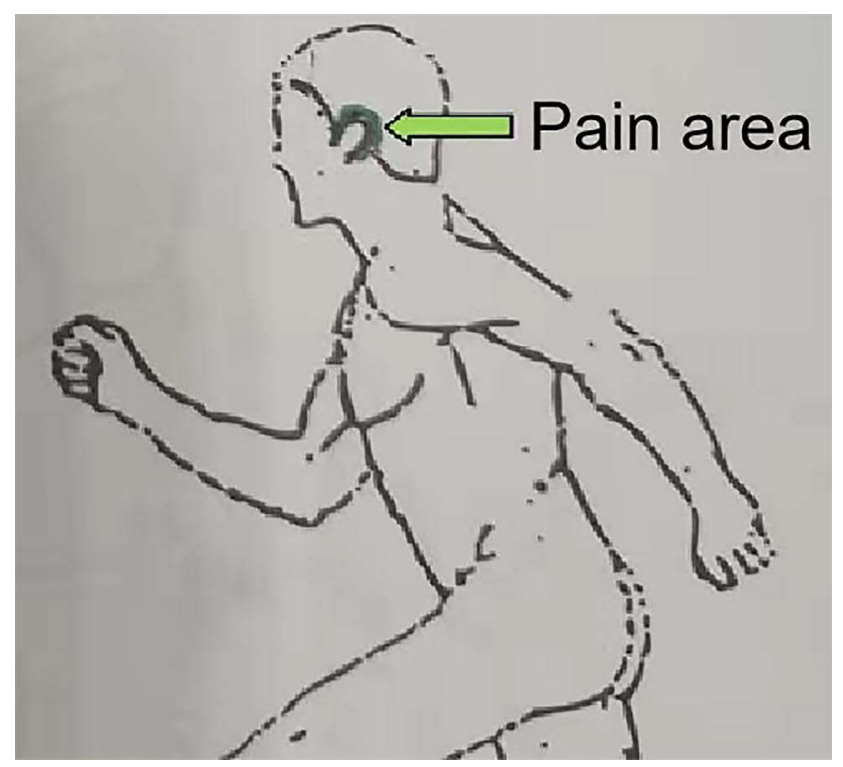

Figure 3 Pain area after PRF treatment of the GAN.

SSS score was still 41, the PHQ-9 score was 5, and the GAD7 score was 4 , as discharged after $7 \mathrm{~d}$ post-op.

\section{Discussion}

In the case, the PRF of the left $\mathrm{C} 2$ dorsal root ganglion and the left superficial cervical nerves under the guidance of DSA was firstly performed for the patient with cervical and occipital PHN in his local hospital. After the first treatment, the postoperative PI-NRS score of the patient was decreased from 8 to 5 , and the pain area was reduced from the left auricle, posterior ear, occipitalia and neck to the left auricle and part of the external ear, and the lesion area was completely numb. However, the residual pain was not relieved despite increasing the dose of opioid as time went on. The NRS score was reduced from 5 to 1 immediately when ultrasound-guided selective PRF treatment of GAN in our hospital, the numbness degree of the lesion area was relieved by $60 \%$, and the pain area was further reduced (Figure 3), so as to improve the sleep and potential co-occurring psychiatric disorders. There were no adverse effects in this original practice. As a result, we confirmed the efficacy, safety and clinical feasibility of ultrasound-guided selective PRF treatment of GAN.

PHN is the most common complication of herpes zoster, and the annual incidence rate is $3.9 \sim 42.0 / 100,000 .{ }^{13}$ It is also a representative of clinically intractable neuropathic pain. In a sense, intractable pain and chronic pain blend together. In the past, it had been reported that the incidence of depression and anxiety is much higher among people with pain, especially chronic pain. In other words, high rates of depression and anxiety have been consistently reported among patients suffering from chronic pain. ${ }^{11,14}$ Kroenke et al, collected 965 randomly sampled patients from consecutive clinic patient to investigate the prevalence, impairment, comorbidity of anxiety disorders in primary care, and they turned out that each disorder was associated with substantial impairment that increased significantly as the number of anxiety disorders increased. ${ }^{15} \mathrm{In}$ fact, they also proved the significance of the SSS for the patients (namely, the process of patients' psychological disorders is from somatization symptoms, anxiety and depression to severe depression). That's why we wanted to do the questionnaires of the PHQ-9, GAD-7, SSS with a patient before and after treatments, these data may be valuable to evaluate the outcome of treatments. The score of the patient in this case before the treatment in our hospital (PHQ-9: 7, GAD-7: 8, SSS: 41) showed a tendency towards anxiety and depression, and moderate somatization disorders clinically, at least, obviously decreased among two of them before discharged, meaning overall improvement in PHN and psychiatric disorders in a way. For a series of diseases bringing chronic pain such as tension headache, migraine, and cancerous pain, the questionnaires mentioned above have been widely used to illustrate the presence and severity of depression or anxiety. ${ }^{11,14-16}$ However, to our knowledge, there has been no report that investigation from these questionnaires has been used in patients with PHN. If the data involving a specific objects of research and intervention measures could been analysed, we might obtain some important information to treat the pain, depression and anxiety of PHN from the statistical perspective. Of course, a scientific experimental design and large sample size are indispensable, perhaps the RF neuroablation of the GAN can be attempted. Briefly, although the treatment of depression and anxiety disorders were not highlighted in this article, it is necessary for us to pay close attention to depression and anxiety disorders with PHN, which helps clinicians choose and administer the appropriate treatment methods by investigating the PHQ-9, the GAD-7, and the SSS.

Regarding the treatment of PHN, in recent years, pulse $\mathrm{RF}$ techniques of the corresponding nerves or nerves root are more and more prevalent in pain department. It is a neuromodulation technique developed on the basis of continuous radiofrequency damage therapy by selectively changing the fiber structure of small myelinated $\mathrm{A} \delta$ and unmyelinated $\mathrm{C}$ pain afferent fiber axons, ${ }^{17}$ so as to inhibit 

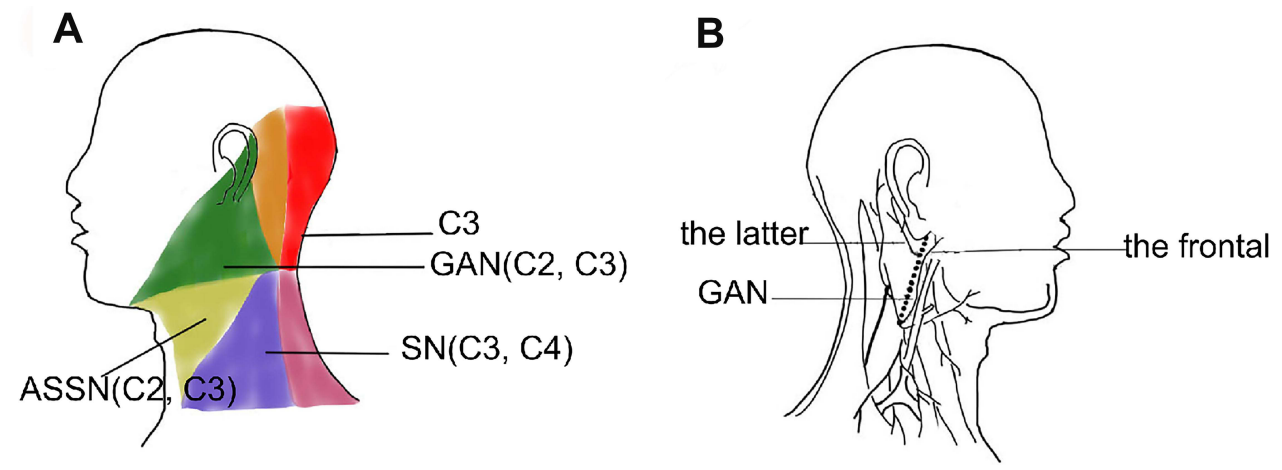

Figure 4 Partial nerve distribution of the ear and neck. (A) C2-C4 sensory nerves distribution of the head and face. (B) The anatomy diagram of the GAN and its branches. Abbreviations: SN, supraclavicular nerve; ASSN, cervical anterior cutaneous nerve.

ectopic spontaneous discharge and interfere with the transmission of nerve impulse. It can achieve the purpose of inhibiting peripheral nerve sensitization by regulating endogenous opioid receptor correlation. ${ }^{18}$ At present, a large number of literature have reported that PRF can achieve great therapeutic effect on the treatment of PHN with different innervations (including dorsal root ganglion, trigeminal nerve, intercostal nerve, etc.), but without the GAN. ${ }^{12,19-21}$ What's more, a report about trying to retrospectively analyse the patients who underwent PRF to zoster related pain (herpes zoster or PHN) proved that early PRF could reduce the use of analgesic and antianxiety drugs and prevent the formation of PHN. ${ }^{12}$

Therefore, in our opinion, the effect of early RF neuroablation in GAN is worth exploring. The GAN is the principal sensory branch of the cervical plexus, consisting of the deep branch of the $\mathrm{C} 2$ nerve and the anterior branch of the C3 nerve, which mainly innervates the antitragus, the tail of the tragus, the lobule of the auricle and segments of the external ear sensation (Figure 4A). Even the sensory innervations of GAN can extend to the tragus spine, tragus and concha. GAN is the largest cutaneous nerve in the cervical plexus. After emanating from the cervical plexus, and arriving at the deep surface of sternocleidomastoid (SCM) muscle, it tilts out and down, passes through the deep fascia to the midpoint of the posterior edge of the SCM muscle and the subcutaneous, then goes up and inside, and finally divides into two or three terminal branches (Figure 4B). Its anatomical position is constant and superficial, and easy to find. ${ }^{22}$ So, the GAN block is commonly used for relieving pain in a variety of surgical operations clinically, including ear hematoma drainage tear repair, etc., and regarded as an anesthesia method for ear cyst resection in the emergency rooms or outpatient treatment rooms. ${ }^{23-25}$ In addition, with the progress of the researchers' understanding of the GAN and accuracy and visualization of ultrasound technology, the patients who are elderly and chronic complications (these diseases may be hazards for intravenous general anesthesia) benefit from ultrasound-guided GAN block for relevant facial surgery, and obtain considerable results in post-operative analgesia by continuous block. ${ }^{26}$ The acupuncture pain the patients feel can reduce closely to $100 \% .{ }^{27}$ In a word, GAN has the advantages of clear ultrasound imaging and is easy to block successfully and completely, which makes the outcome of blocking technique significantly effective and safe with fewer complications related to puncture. ${ }^{27}$ All of relevant contents indicate theoretical and practical basis for RF neuroablation occurring on GAN, and lead us to select it.

\section{Conclusion}

To our knowledge, there was rarely report on the effect of PRF treatment of GAN in PHN patients around the world. We team attempted successfully ultrasound-guided selective PRF treatment of GAN for the first time. The treatment method is safe, effective, applicable and feasible, which can provide more prospective therapy way for PHN in the head and neck, even probably for GAN neuralgia, and be worthy of clinical promotion. In addition, PHQ-9, GAD-7 and SSS might be meaningful to evaluate the efficacy of intractable PHN. The limitation of this paper is that only a sample size meeting the standard is observed and lacks of a follow-up record and data of the post-treatment. We therefore expect more researchers and scholars to test and verify the views elaborated in this case report by expanding the sample size. 


\section{Ethics}

The research was conducted ethically in accordance with the Helsinki Declaration and approved by the Biomedical Research Ethics Committee of the Affiliated Hospital of Zunyi Medical University (No. KLL-2021-007). The patient provided written consent to publish this case, including the use of ultrasound images.

\section{Acknowledgment}

Thanks to the development and application of the new technology, GAN block in auriculotemporal surgery under the ultasound-guided in the Affiliated Hospital of Zunyi Medical University, it provided practical basis for this case and a better therapeutic plan.

\section{Author Contributions}

All authors made substantial and significant contributions to conception and design, acquisition or analysis and interpretation of data, took part in drafting, revising and polishing the article critically for important intellectual content. All authors gave final approval of the version to be published, and have agreed on the journal to which the article has been submitted and to be accountable for all aspects of the work. Ting-Hua Wang and Liu-Lin Xiong are regarded as cocorrespondence authors.

\section{Disclosure}

The authors declared that they have no conflicts of interest for this work.

\section{References}

1. Koshy E, Mengting L, Kumar H, Jiabo W. Epidemiology, treatment and prevention of herpes zoster: a comprehensive review. Indian J Dermatol Venereol Leprol. 2018;84(3):251-262. doi:10.4103/ijdvl. IJDVL_1021_16

2. Sampathkumar P, Drage LA, Martin DP. Herpes zoster (shingles) and postherpetic neuralgia. Mayo Clin Proc. 2009;84(3):274-280. doi:10.4065/84.3.274

3. Pearce JM. Post herpetic neuralgia. J Neurol Neurosurg Psychiatry. 2005;76(4):572. doi:10.1136/jnnp.2004.047381

4. Jeon Y, Kim S. Treatment of great auricular neuralgia with real-time ultrasound-guided great auricular nerve block: a case report and review of the literature. Medicine. 2017;96(12):e6325. doi:10.1097/ MD.0000000000006325

5. Zhenhe L, Zhijian F, Chen J. Radiofrequency Analgesic Therapy. 2nd ed. Zhengzhou: Henan science and technology press; 2019:68.

6. Duvall JR, Garza I, Kissoon NR, Robertson CE. Great auricular neuralgia: case series. Headache. 2020;60(1):247-258. doi:10.1111/ head. 13690
7. Farrar JT, Young JP, LaMoreaux L, Werth JL, Poole MR. Clinical importance of changes in chronic pain intensity measured on an 11-point numerical pain rating scale. Pain. 2001;94(2):149-158. doi:10.1016/S0304-3959(01)00349-9

8. Liu S, Liu Y, Liu Y. Somatic symptoms and concern regarding COVID-19 among Chinese college and primary school students: a cross-sectional survey. Psychiatry Res. 2020;289:113070. doi:10.1016/j.psychres.2020.113070

9. Spitzer RL, Kroenke K, Williams JB, Löwe B. A brief measure for assessing generalized anxiety disorder: the GAD-7. Arch Intern Med. 2006;166(10):1092-1097. doi:10.1001/ archinte.166.10.1092

10. American Psychiatric Association. Diagnostic and Statistical Manual of Mental Disorders. 4th ed. Washington, DC: American Psychiatric Association; 1994.

11. Feingold D, Brill S, Goor-Aryeh I, Delayahu Y, Lev-Ran S. Depression and anxiety among chronic pain patients receiving prescription opioids and medical marijuana. Affect Disord. 2017;218:1-7. doi:10.1016/j.jad.2017.04.026

12. Kim K, Jo D, Kim E. Pulsed radiofrequency to the dorsal root ganglion in acute herpes zoster and postherpetic neuralgia. Pain Physician. 2017;20(3):411-418. doi:10.36076/ppj.2017.E418

13. Van Hecke O, Austin SK, Khan RA, Smith BH, Torrance N. Neuropathic pain in the general population: a systematic review of epidemiological studies. Pain. 2014;155(4):654-662. doi:10.1016/j. pain.2013.11.013

14. Neria Y, Besser A, Kiper D, Westphal M. A longitudinal study of posttraumatic stress disorder, depression, and generalized anxiety disorder in Israeli civilians exposed to war trauma. Journal of Traumatic Stress. 2010;23(3):322-330. doi:10.1002/jts.20522

15. Kroenke K, Spitzer RL, Williams JB, Monahan PO, Löwe B. Anxiety disorders in primary care: prevalence, impairment, comorbidity, and detection. Ann Intern Med. 2007;146(5):317-325. doi:10.7326/00034819-146-5-200703060-00004

16. Seo JG, Park SP. Validation of the Generalized Anxiety Disorder-7 (GAD-7) and GAD-2 in patients with migraine. $J$ Headache pain. 2015;16:97. doi:10.1186/s10194-015-0583-8

17. Jia Z, Ren H, Li Q, Ji N, Luo F. Pulsed radiofrequency reduced neuropathic pain behavior in rats associated with upregulation of GDNF expression. Pain Physician. 2016;19(2):49. doi:10.36076/ $\mathrm{ppj} / 2016.19 .49$

18. Moffett J, Fray LM, Kubat NJ. Activation of endogenous opioid gene expression in human keratinocytes and fibroblasts by pulsed radiofrequency energy fields. J Pain Res. 2012;5:347-357. doi:10.2147/ JPR.S35076

19. Kim ED, Lee YI, Park HJ. Comparison of efficacy of continuous epidural block and pulsed radiofrequency to the dorsal root ganglion for management of pain persisting beyond the acute phase of herpes zoster. PLoS One. 2017;12(8):e0183559. doi:10.1371/journal. pone. 0183559

20. Pi ZB, Lin H, He GD, Cai Z, Xu XZ. Randomized and controlled prospective trials of Ultrasound-guided spinal nerve posterior ramus pulsed radiofrequency treatment for lower back post-herpetic neuralgia. Clin Ter. 2015;166(5):301-305.

21. Makharita MY, El Bendary HM, Sonbul ZM, Ahmed SES, Latif MA. Ultrasound-guided pulsed radiofrequency in the management of thoracic postherpetic neuralgia: a randomized, double-blinded, controlled trial. Clin J Pain. 2018;34(11):1017-1024. doi:10.1097/AJP.000000 0000000629

22. Wang L, Xin Y, Dang R. Applied anatomy of the great auricular nerve. Anat J. 1995;18(6):499-500. 
23. Selekler M, Kutlu A, Uçar S, Almaç A. Immediate response to greater auricular nerve blockade in red ear syndrome. Cephalalgia. 2009;29:478-479. doi:10.1111/j.1468-2982.2008.01756.x

24. Herring AA, Stone MB, Frenkel O, Chipman A, Nagdev AD. The ultrasound-guided superficial cervical plexus block for anesthesia and analgesia in emergency care settings. Am Emerg Med. 2012;30:1263-1267.

25. Gleeson AP, Gray AJ. Management of retained ear-rings using an ear block. Emergency Medicine Journal. 1995;12:199-201. doi:10.1136/ emj.12.3.199
26. Ritchie MK, Wilson CA, Grose BW, Ranganathan P, Howell SM, Ellison MB. Ultrasound-guided greater auricular nerve block as sole anesthetic for ear surgery. Clin Pract. 2016;6(2):856. doi:10.4081/ cp. 2016.856

27. Ellison MB, Howell S, Heiraty P, Wilson C, Shepherd J, Ellison PR. A novel approach to postoperative ear pain-greater auricular nerve block catheter: a case report. A A Pract. 2020;14(1):21. doi:10.1213/ XAA.0000000000001134

\section{Publish your work in this journal}

The Journal of Pain Research is an international, peer reviewed, open access, online journal that welcomes laboratory and clinical findings in the fields of pain research and the prevention and management of pain. Original research, reviews, symposium reports, hypothesis formation and commentaries are all considered for publication. The manuscript

Submit your manuscript here: https://www.dovepress.com/journal-of-pain-research-journa management system is completely online and includes a very quick and fair peer-review system, which is all easy to use. Visit http:// www.dovepress.com/testimonials.php to read real quotes from published authors. 